EPJ Web of Conferences 112,02016 (2016)

DOI: $10.1051 /$ epjconf/201611202016

(C) Owned by the authors, published by EDP Sciences, 2016

\title{
JIMWLK and beyond: From concepts to observables
}

\author{
Heribert Weigert ${ }^{1}$, Tuomas Lappi ${ }^{2,4}$, Andrecia Ramnath ${ }^{2}$, and Kari Rummukainen ${ }^{3,4}$ \\ ${ }^{1}$ Department of Physics, University of Cape Town, Private Bag X3, Rondebosch 7701, South Africa \\ ${ }^{2}$ Department of Physics, University of Jyväskylä, P.O. Box 35, 40014 University of Jyväskylä, Finland \\ ${ }^{3}$ Department of Physics, University of Helsinki, 00014 University of Helsinki, Finland \\ ${ }^{4}$ Helsinki Institute of Physics, P.O. Box 64, 00014 University of Helsinki, Finland
}

\begin{abstract}
The Color Glass Condensate and its associated evolution equation, the JIMWLK equation have applications to many observables far beyond totally inclusive observables. The phenomenology is so rich that little has been done to explore beyond scaling behavior of correlators. We show first examples that exemplify the considerations necessary to access additional information both experimentally and theoretically and demonstrate that the Wilson line correlators appearing throughout make it imperative to consistently take into account that one is dealing with correlators of group elements and demonstrate how this imposes physical and phenomenological constraints. Similar considerations apply also to jet observables at finite $N_{c}$.
\end{abstract}

\section{Introduction}

Over the last decade, the notion of the Color Glass Condensate and its associated evolution equation, the JIMWLK equation, has become a mature tool to quantitatively describe QCD cross sections and the initial state entering heavy ion collisions at high energy. The key ingredient to its derivation is the observation that in a suitably asymmetric high energy collision, where a Fock space expansion of a dilute target in terms of its parton constituents is meaningful the interaction of these constituents with the target gluon field is fully eikonal. Light like Wilson lines along the projectile constituent trajectories then fully summarize the interaction; their correlators fully determine the cross sections. Of course, Wilson lines appear in many other QCD observables in which a separation of hard and soft degrees of freedom is possible. A well established example are non-global jet observables [1] where evolution inside predefined jet cones maps directly onto the structure of the JIMWLK equation (e.g. [2]) via a stereographic projection [3], a fact that has been exploited to infer the NLO contributions of JIMWLK from their jet counterparts [4]. The physics discussions of CGC applications at the leading order all center on scaling phenomena, the behavior of the saturation scale $Q_{s}(Y)$ and how it impacts the total cross section for deep inelastic scattering and similarly inclusive phenomena such as total multiplicities in heavy ion collisions. In this contribution we attempt to broaden that picture somewhat and give a glimpse at a richer set of accessible phenomena that will show radically different behavior. To do so one needs a systematic way of including degrees of freedom beyond the pomeron. Naturally, the first glimpse at qualitatively new 
behavior comes from a consistent inclusion of odderon degrees of freedom. Since the gist of this is already present at leading order (LO) we will keep the discussion on this level- it does generalize to full next to leading order (NLO).

\section{Observables, hierarchies, and parametrizations}

The simplest example for Wilson line correlators in cross sections is the expression for the DIS total cross section at small $x$ to leading order. Its diagrammatic expression reads

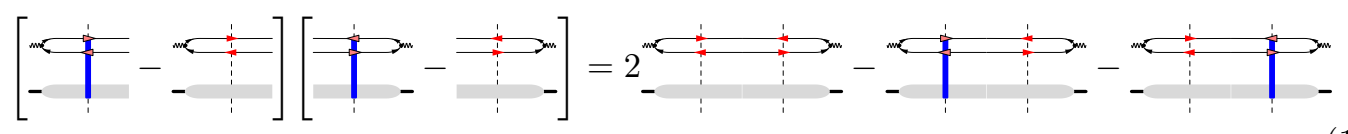

The Wilson line correlators appear in the last two terms. Since this is an expression for the total cross section they explicitly combine into the real part of this correlator

$$
\left\langle\operatorname{tr}\left(U_{\boldsymbol{x}} U_{\boldsymbol{y}}^{\dagger}\right)\right\rangle(Y)+\left\langle\operatorname{tr}\left(U_{\boldsymbol{x}}^{\dagger} U_{\boldsymbol{y}}\right)\right\rangle(Y)=2\left\langle\operatorname{Re} \operatorname{tr}\left(U_{\boldsymbol{x}} U_{\boldsymbol{y}}^{\dagger}\right)\right\rangle(Y)
$$

Average displayed is explicitly $Y=\ln (1 / x)$ dependent and to be understood as a QCD average in the presence of the target wave function.

The Wilson line correlator itself does have both a real and an imaginary part although one needs a more exclusive observable to probe it. A suitable example is the Single Transverse Spin Asymmetry (STSA), the Sivers' function, for which Kovchegov and Sievert [5] have suggested a new mechanism at small $x$ that is triggered by this imaginary part. Diagrammatically

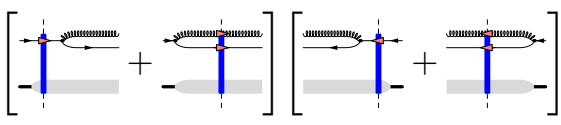

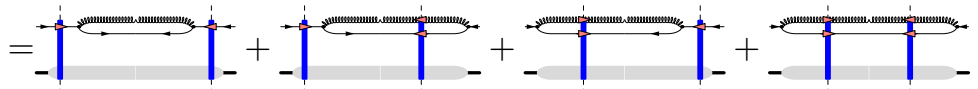

where the momentum and spin of the quark in the final state is tagged and the color in the initial state is summed over. Tagging the quark momentum leads to different coordinates on the corresponding Wilson lines in amplitude and complex conjugate amplitude. The gluon momentum is integrated over so that the gluon Wilson line in the last term cancels between the two sides of the cut. As a result the color correlators in Eq. (3) -in term by term correspondence to the diagrams- are

$$
\mathcal{I}^{(q)}=\left\langle\frac{\operatorname{tr}\left(U_{\boldsymbol{z}} U_{\boldsymbol{y}}^{\dagger}\right)}{N_{c}}-\frac{1}{d_{A}} \tilde{U}_{\boldsymbol{x}}^{a b} \operatorname{tr}\left(t^{a} U_{\boldsymbol{z}} t^{b} U_{\boldsymbol{w}}^{\dagger}\right)-\frac{1}{d_{A}} \tilde{U}_{\boldsymbol{x}}^{a b} \operatorname{tr}\left(t^{a} U_{\boldsymbol{u}} t^{b} U_{\boldsymbol{y}}^{\dagger}\right)+\frac{\operatorname{tr}\left(U_{\boldsymbol{u}} U_{\boldsymbol{w}}^{\dagger}\right)}{N_{c}}\right\rangle
$$

The STSA is driven by the contributions that are anti-symmetric under exchange of the quark and anti-quark coordinates $\boldsymbol{z} \leftrightarrow \boldsymbol{y}$ and thus the imaginary part of, for example the first term.

Focusing on the the dipole correlator, we parametrize this complex number in terms of two real degrees of freedom

$$
S_{\boldsymbol{x} \boldsymbol{y}}(Y):=\left\langle\operatorname{tr}\left(U_{\boldsymbol{x}} U_{\boldsymbol{y}}^{\dagger}\right)\right\rangle(Y) / N_{c}=1-P_{\boldsymbol{x} \boldsymbol{y}}(Y)+i O_{\boldsymbol{x} \boldsymbol{y}}(Y)=e^{-C_{f}\left(\mathcal{P}_{\boldsymbol{x} \boldsymbol{y}}+i \mathcal{O}_{\boldsymbol{x} \boldsymbol{y}}\right)(Y)}
$$

either directly through real and imaginary parts $\left(1-P_{\boldsymbol{x} y}\right.$ and $O_{\boldsymbol{x} \boldsymbol{y}}$ respectively) or exponentially via a logarithmic modulus and phase $\left(\mathcal{P}_{\boldsymbol{x} \boldsymbol{y}}\right.$ and $\mathcal{O}_{\boldsymbol{x} \boldsymbol{y}}$ respectively). Noting that complex 
conjugation simply swaps the coordinates on $S, S_{\boldsymbol{x} \boldsymbol{y}}^{*}=S_{\boldsymbol{y} \boldsymbol{x}}$, implies that $P$ and $\mathcal{P}$ are symmetric, while $O$ and $\mathcal{O}$ are anti-symmetric under the exchange of $\boldsymbol{x}$ and $\boldsymbol{y}$. This symmetry property links them to the pomeron and odderon respectively.

The three point function of the form $\tilde{U}_{\boldsymbol{z}}^{a b} \operatorname{tr}\left(t^{a} U_{\boldsymbol{x}} t^{b} U_{\boldsymbol{y}}^{\dagger}\right)$ in Eq. (4) -it also appears in the evolution equation of the $q \bar{q}$ dipole operator- is slightly more complicated but intimately related to the $q \bar{q}$ and $g g$ dipole operators in different coincidence limits. Schematically

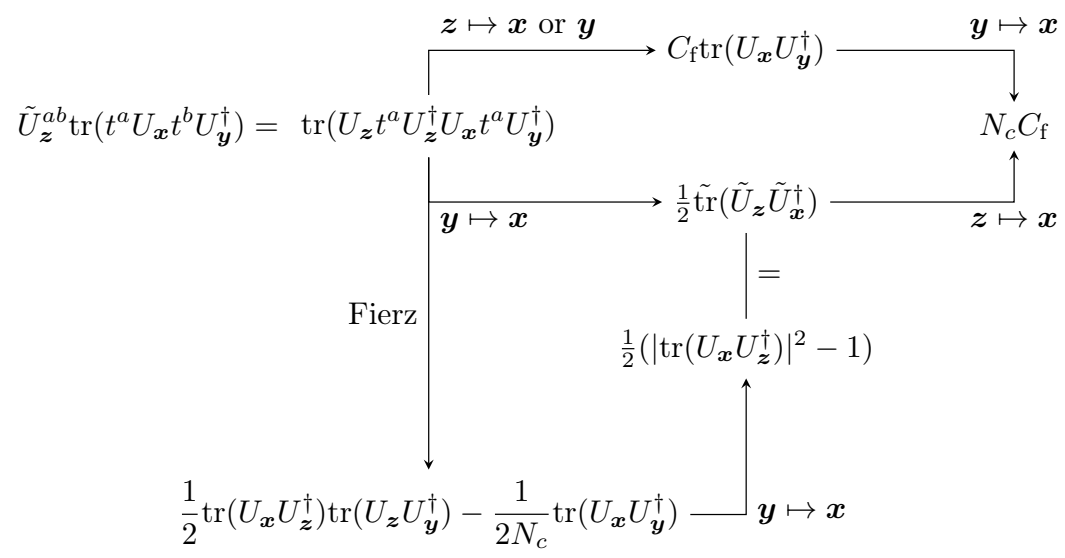

It is well known that the JIMWLK equation, already at LO, is equivalent to infinite coupled hierarchies of evolution equations for Wilson line correlators. It is worth emphasizing that it governs the $Y$ dependence of both real and imaginary parts of all of these correlators although many applications are blind to the imaginary parts: It turns out that they may be set to zero consistently within the initial conditions of the evolution equation if not needed phenomenologically.

A general parametrization that manifestly respects the relationships in (6) is a generalization of the Gaussian truncation used already in [6-9] where we truncate at the level of three point functions:

$$
\begin{aligned}
\langle\ldots\rangle(Y)=\left\langleP _ { Y } \operatorname { e x p } \left\{\int_{Y_{0}}^{Y} d y[\right.\right. & -\frac{1}{2} \int_{\boldsymbol{u} \boldsymbol{v}} G_{\boldsymbol{u} \boldsymbol{v}}(y) i \nabla_{\boldsymbol{u}}^{a} i \nabla_{\boldsymbol{v}}^{a} \\
& \left.\left.\left.-\frac{1}{3 !} \int_{\boldsymbol{u} \boldsymbol{v} \boldsymbol{w}} G_{\boldsymbol{u} \boldsymbol{v} \boldsymbol{w}}(y) d^{a b c} i \nabla_{\boldsymbol{u}}^{a} i \nabla_{\boldsymbol{v}}^{b} i \nabla_{\boldsymbol{w}}^{c}+\text { etc. }\right]\right\} \ldots\right\rangle\left(Y_{0}\right) .
\end{aligned}
$$

This can be generalized systematically at any n-point level, the number of singlets of $n$ objects in the adjoint representation determines the number of $n$-point functions appearing in the $Y$-ordered exponential. For convenience we choose $G_{\boldsymbol{u} \boldsymbol{u}}=0$ and $G_{\boldsymbol{u} \boldsymbol{u} \boldsymbol{u}}=0$ and do not display the three point function associated with $f^{a b c}$ as it does not enter the correlators discussed here.

Eq. (7) induces explicit expressions for all correlators in Eqns. (2) and (4), namely

$$
\begin{aligned}
& \frac{1}{N_{c}}\left\langle\operatorname{tr}\left(U_{\boldsymbol{x}} U_{\boldsymbol{y}}^{\dagger}\right)\right\rangle=e^{-C_{f}(\mathcal{P}+i \mathcal{O})_{\boldsymbol{x} y}} \\
& \frac{1}{2 N_{c} C_{f}}\left\langle U_{\boldsymbol{z}}^{a b} \operatorname{tr}\left(t^{a} U_{\boldsymbol{x}} t^{b} U_{\boldsymbol{y}}^{\dagger}\right)\right\rangle=e^{-\left\{\left[\frac{N_{c}}{2}\left((\mathcal{P}+i \mathcal{O})_{\boldsymbol{x} \boldsymbol{z}}+(\mathcal{P}+i \mathcal{O})_{\boldsymbol{z} \boldsymbol{y}}\right)-(\mathcal{P}+i \mathcal{O})_{\boldsymbol{x} \boldsymbol{y}}\right)-C_{\mathrm{f}}(\mathcal{P}+i \mathcal{O})_{\boldsymbol{x} \boldsymbol{y}}\right\}(Y)}
\end{aligned}
$$


where

$\mathcal{P}_{\boldsymbol{x} \boldsymbol{y}}:=\int^{Y} d y\left(G_{\boldsymbol{x} \boldsymbol{y}}-\frac{1}{2}\left(G_{\boldsymbol{x} \boldsymbol{x}}+G_{\boldsymbol{y} \boldsymbol{y}}\right)=\mathcal{P}_{\boldsymbol{y} \boldsymbol{x}} \quad i \mathcal{O}_{\boldsymbol{x} \boldsymbol{y}}:=\frac{C_{d}}{4} \int^{Y} d y\left(G_{\boldsymbol{y} \boldsymbol{x} \boldsymbol{x}}-G_{\boldsymbol{y} \boldsymbol{y} \boldsymbol{x}}\right)=-i \mathcal{O}_{\boldsymbol{y} \boldsymbol{x}}\right.$

Strikingly, the observables considered here do not allow access to $G_{\boldsymbol{u v w}}$ with all three coordinates independent! The effective two point functions however do show the required symmetry properties; one obtains a consistent gauge invariant truncation of the associated Balitsky hierarchy with the evolution equation

$$
\frac{d}{d Y} \mathcal{G}_{\boldsymbol{x} \boldsymbol{y}}(Y)=\frac{\alpha_{s}}{\pi^{2}} \int d^{2} z K_{\boldsymbol{x} \boldsymbol{z} \boldsymbol{y}}\left(1-e^{-\frac{N_{\mathrm{c}}}{2}\left[\mathcal{G}_{\boldsymbol{x} \boldsymbol{z}}+\mathcal{G}_{\boldsymbol{z} \boldsymbol{y}}-\mathcal{G}_{\boldsymbol{x} \boldsymbol{y}}\right](Y)}\right) \quad \mathcal{G}_{\boldsymbol{x} \boldsymbol{y}}(Y)=(\mathcal{P}+i \mathcal{O})_{\boldsymbol{x} \boldsymbol{y}}(Y)
$$

which obviously couples real and imaginary parts. Eq. (11) generalizes the the large $N_{c}$ results of $[10,11]$. Curiously, Eq. (11) differs from the BK equation for the total cross section in the Gaussian truncation only through a non-vanishing imaginary part $i \mathcal{O} \neq 0$ (this is one of the differences between our treatment and the discussion in $[10,11])$. Indeed $i \mathcal{O}=0$ and $\mathcal{P} \in[0,1]$ is a consistent solution to this equation which leads to a successful phenomenology for HERA data at small $x$ [9]: If the initial condition for $\mathcal{G}$ is real, the equation never generates an imaginary part. This behavior is numerically stable as can be seen from analyzing the coupled equation for the real and imaginary parts explicitly:

$$
\begin{aligned}
\frac{d}{d Y} \mathcal{P}_{\boldsymbol{x} \boldsymbol{y}}(Y) & =\frac{\alpha_{s}}{\pi^{2}} \int d^{2} z K_{\boldsymbol{x} \boldsymbol{z} \boldsymbol{y}}\left(1-e^{-\frac{N_{c}}{2}\left[\mathcal{P}_{\boldsymbol{x} \boldsymbol{z}}+\mathcal{P}_{\boldsymbol{z} \boldsymbol{y}}-\mathcal{P}_{\boldsymbol{x} \boldsymbol{y}}\right](Y)} \cos \left(\frac{N_{\mathrm{c}}}{2}\left[\mathcal{O}_{\boldsymbol{x} \boldsymbol{z}}+\mathcal{O}_{\boldsymbol{z} \boldsymbol{y}}-\mathcal{O}_{\boldsymbol{x} \boldsymbol{y}}\right](Y)\right)\right) \\
\frac{d}{d Y} \mathcal{O}_{\boldsymbol{x} \boldsymbol{y}}(Y) & =\frac{\alpha_{s}}{\pi^{2}} \int d^{2} z K_{\boldsymbol{x} \boldsymbol{z} \boldsymbol{y}}\left(e^{-\frac{N_{c}}{2}\left[\mathcal{P}_{\boldsymbol{x} \boldsymbol{z}}+\mathcal{P}_{\boldsymbol{z} \boldsymbol{y}}-\mathcal{P}_{\boldsymbol{x} \boldsymbol{y}}\right](Y)} \sin \left(\frac{N_{\mathrm{c}}}{2}\left[\mathcal{O}_{\boldsymbol{x} \boldsymbol{z}}+\mathcal{O}_{\boldsymbol{z} \boldsymbol{y}}-\mathcal{O}_{\boldsymbol{x} \boldsymbol{y}}\right](Y)\right)\right)
\end{aligned}
$$

The odderon contribution will naturally shrink no matter which initial condition is used for it.

The choice of a such an initial condition for the pair of $\mathcal{P}$ and $\mathcal{O}$, one that is physically meaningful, needs some thought. At short distance, light cone perturbation theory leads to $\mathcal{P}_{\boldsymbol{x} \boldsymbol{y}} \propto|\boldsymbol{x}-\boldsymbol{y}|^{2}$, while $\mathcal{O}_{\boldsymbol{x} \boldsymbol{y}} \propto|\boldsymbol{x}-\boldsymbol{y}|^{3}$ [10], but the symmetry properties imposed by complex conjugation and evidenced in Eq. (10) require the presence of an additional transverse vector $\hat{s}$ - to be able to see a scalar odderon function in an experiment one needs to break rotational symmetry in the transverse plane such as is done by measuring a spin asymmetry in STSA. We thus expect a short distance behavior of the form

$$
\mathcal{P}_{\boldsymbol{x} \boldsymbol{y}} \propto \boldsymbol{r}^{2} Q_{0}^{2}, \quad \mathcal{O}_{\boldsymbol{x} \boldsymbol{y}} \propto|\boldsymbol{r}|^{3} \kappa Q_{0}^{2} \hat{\boldsymbol{r}} \cdot \hat{\boldsymbol{s}} \quad \text { where } \quad \boldsymbol{r}=\boldsymbol{x}-\boldsymbol{y} .
$$

$Q_{0}$ is of the order of $Q_{s}\left(Y_{0}\right)$, the well know saturation scale of the initial condition, but $\kappa$, the scale ratio relating the scales of the two contributions, is not yet constrained.

A qualitative and even semi-quantitative constraint comes from an elementary group theoretical condition on the dipole correlator $\operatorname{tr}\left(U_{\boldsymbol{x}} U_{\boldsymbol{y}}^{\dagger}\right)$. As an element of $\mathrm{SU}\left(N_{c}\right)$ any Wilson line configuration $U_{\boldsymbol{x}} U_{\boldsymbol{y}}^{\dagger}$ has eigenvalues on the unit circle $e^{i \phi_{i}}$ which are subject to the determinant condition $\sum_{i=1}^{N_{c}} \phi_{i}=2 \pi n ; n \in \mathbb{Z}$, so that the trace of any individual configuration can 

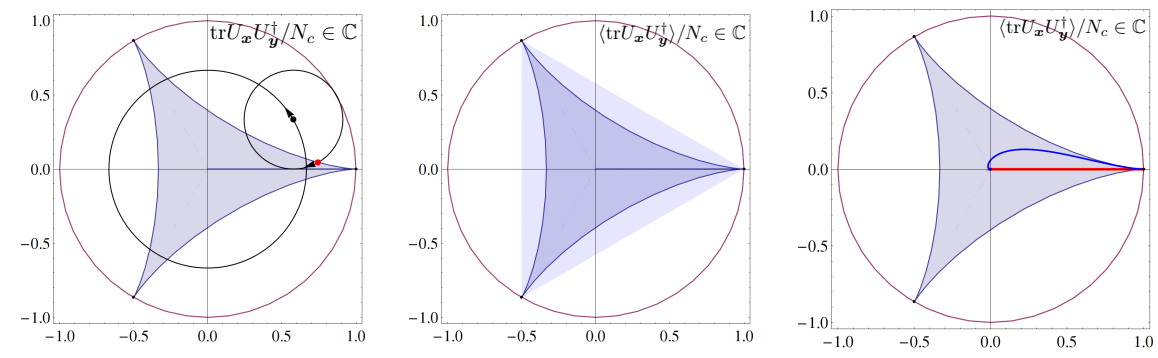

Figure 1. Left: The trace of a given group element of SU(3) such as a given dipole configuration $\operatorname{tr}\left(U_{\boldsymbol{x}} U_{\boldsymbol{y}}^{\dagger}\right) / N_{c}$ falls into the hypocycloid shown in grey. Its boundary is traced out by a point on the smaller circle shown in red rolling counter-clockwise inside the outer circle. Middle: The expectation value or average of such configurations $\left\langle\operatorname{tr}\left(U_{\boldsymbol{x}} U_{\boldsymbol{y}}^{\dagger}\right)\right\rangle(Y) / N_{c}$ is slightly less constrained: It may fall outside the hypocycloid but must remain inside a polygon connecting the cusps. Right: Perturbative initial conditions for real and imaginary parts of $\left\langle\operatorname{tr}\left(U_{\boldsymbol{x}} U_{\boldsymbol{y}}^{\dagger}\right)\right\rangle(Y) / N_{c}$ for ensembles with or without a net odderon (blue and red lines respectively). The blue line represents the extreme case assuming there is no non-perturbative mechanism to pull the average outside the hypocycloid.

be parametrized as

$$
\operatorname{tr}\left(U_{\boldsymbol{x}} U_{\boldsymbol{y}}^{\dagger}\right) / N_{c}=\frac{1}{N_{c}}\left(\sum_{i=1}^{N_{c}-1} e^{i \phi_{i}}+e^{-i \sum_{i=1}^{N_{c}-1} \phi_{i}}\right)
$$

with $N_{c}-1$ distinct phases $\phi_{i}$, all $\boldsymbol{x}$ and $\boldsymbol{y}$ dependent. (15) falls into a hypocycloid in the complex plane with $N_{c}$ cusps, each representing one of the center elements of $\mathrm{SU}\left(N_{c}\right)$ as shown for $N_{c}=3$ in Fig 1. (This is a textbook result, see [12] for a recent discussion.) The expectation value or average of such configurations, $\left\langle\operatorname{tr}\left(U_{\boldsymbol{x}} U_{\boldsymbol{y}}^{\dagger}\right)\right\rangle(Y) / N_{c}$, is slightly less constrained. It may fall outside the hypocycloid but must remain inside a polygon connecting the cusps. Note, however, that the cusps corresponding to the nontrivial center elements (at $e^{ \pm 2 \pi i / 3}$ for $N_{c}=3$ ) also correspond to non-perturbative configurations - only the vicinity of the unit element corresponds to a short distance correlator. Hence, one needs a nonperturbative mechanism to push the average outside the hypocycloid. In either case, this puts severe constraints on an initial condition that extrapolates the short distance limit through exponentiation in the form $\left(Q_{0}\right.$ is of the order of $Q_{s}\left(Y_{0}\right)$, the real part is nothing but the Golec-Biernat Wüsthoff model)

$$
\left\langle\operatorname{tr}\left(U_{\boldsymbol{x}} U_{\boldsymbol{y}}^{\dagger}\right)\right\rangle\left(Y_{0}\right) / N_{c}=e^{-r^{2} Q_{0}^{2}+i \kappa r^{3} Q_{0}^{3} \hat{\boldsymbol{r}} \cdot \hat{\boldsymbol{s}}}
$$

For the first case $\kappa \leq 1 / 3$ while for the second we get an approximate bound of $\kappa \leq .98$. The first case is shown in Fig. 1, right panel. Real and imaginary parts of this expression are shown in Fig. 2 for both extreme cases. Real and imaginary parts show modulation only near $Q_{s}\left(Y_{0}\right)$ and thus provide perturbatively consistent starting points for evolution in a calculation where $Q_{s}\left(Y_{0}\right)$ is assumed to be in the perturbative domain. Note that the size of the odderon peak (the maximum of imaginary parts shown in red in Fig. 2) is severely limited by the bounds on $\kappa$. This is very different from the BFKL odderon in which there is only a phenomenological reason to assume a small odderon contribution in the initial condition. 



Figure 2. Perturbatively motivated initial conditions for both real and imaginary parts (blue and red respectively) of $\left\langle\operatorname{tr}\left(U_{\boldsymbol{x}} U_{\boldsymbol{y}}^{\dagger}\right)\right\rangle\left(Y_{0}\right) / N_{c}=e^{-r^{2} Q_{0}^{2}+i \kappa r^{3} Q_{0}^{3} \hat{\boldsymbol{r}} \cdot \hat{\boldsymbol{s}}}$ at $\hat{\boldsymbol{r}} \cdot \hat{\boldsymbol{s}}=1$ assuming no non-perturbative mechanism to drive the correlator outside the hypocycloid (left) and relaxing this condition (right). In both cases real and imaginary parts show modulation only near $Q_{s}\left(Y_{0}\right)$.

In the non-linear case both the pomeron and odderon are subject to group constraints and severely limited in magnitude.

\section{Qualitative simulation results}

The easiest way to study the qualitative energy behavior of the coupled pomeron and odderon configurations is a full Langevin simulation of the JIMWLK equation as first done in [13] and developed further for example in $[14,15]$. This simulation does not even require a modification of the evolution code: The only ingredient requiring modification is the initial condition. One must start from an ensemble in which, on average, the imaginary part of $\left\langle\operatorname{tr}\left(U_{\boldsymbol{x}} U_{\boldsymbol{y}}^{\dagger}\right)\right\rangle\left(Y_{0}\right) / N_{c}$ is nonzero. As discussed above this requires that rotational invariance is broken. We have implemented this by distorting a symmetric initial condition to show a bias along one of the coordinate axes of the transverse plane.

Fig. 3 shows the result for a pure pomeron ensemble in the top row and an ensemble with an initial odderon admixture in the bottom row, clearly demonstrating that any odderon admixture (the imaginary part) is erased quickly and efficiently with only minimal feedback into the behavior of the pomeron (the real part). The behavior of the two is strikingly different: While the pomeron shows clear scaling behavior in both cases, the odderon (if present in the initial condition) does not scale - it is erased in place, the maximum falls to zero exponentially without moving much as $Y$ increases.

\section{Conclusions}

The fact that JIMWLK evolution deals with observables entirely expressed in terms of Wilson line correlators - a feature shared with a large set of jet evolution observables- gives a strong handle on both structure and possible initial conditions of such correlators. Coincidence limits reveal simpler correlators with fewer Wilson lines appearing in any given n-point function, the web of relationships for the $q \bar{q} g$ correlator shown in Eq. (6) is but a simple example for 


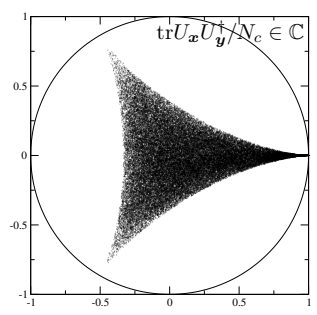



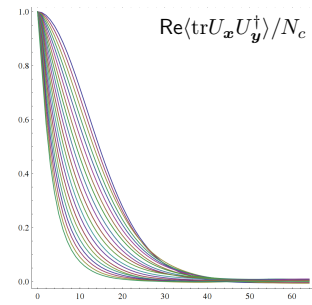

$|\boldsymbol{x}-\boldsymbol{y}| \Lambda$

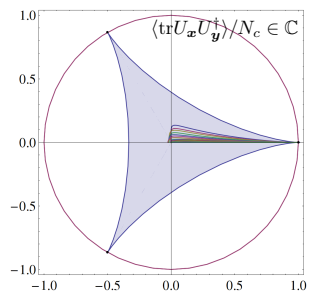

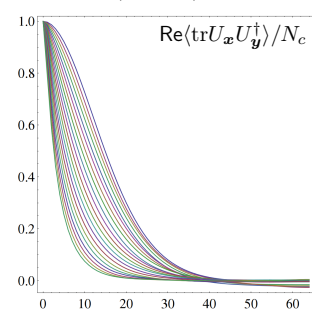

$|\boldsymbol{x}-\boldsymbol{y}| \Lambda$



$|\boldsymbol{x}-\boldsymbol{y}| \Lambda$



$|\boldsymbol{x}-\boldsymbol{y}| \Lambda$

Figure 3. Leading order Langevin simulations with curves for different $Y$ values. The top row shows evolution with zero odderon in the initial condition, the bottom row shows an ensemble with an odderon admixture in the initial condition. The first colum shows a scatterplot of individual traces from the simulation to its right. The non-perturbative cusps are not populated. The second column shows the average $\left\langle\operatorname{tr} U_{\boldsymbol{x}} U_{\boldsymbol{y}}^{\dagger}\right\rangle / N_{c}$ in the complex plane, $|\boldsymbol{x}-\boldsymbol{y}|$ grows as one follows any individual curve from 1 to 0 in $\mathbb{C}$. The third column shows the real parts (evolution moves these from "right to left"), the pomeron, and the fourth column provides the imaginary parts, the odderon (evolution moves these downwards). Both pomeron and odderon contributions are plotted as a function of $|\boldsymbol{r}|=|\boldsymbol{x}-\boldsymbol{y}|$ in arbitrary units - units can only be determined through a fit to data. Real and imaginary parts show different qualitative behavior: while the real parts exhibit scaling behavior the imaginary parts do not move, instead they are erased in place.

the general case. To consistently truncate the hierarchies of evolution equations on the $n$ point level, one needs a parametrization that respects the coincidence limits the Wilson line correlators satisfy by construction. The examples shown give a first glimpse at a classification of correlators of Wilson lines, be it at small $x$ or for jet observables, that is quite analogous to the discussion of Generalized Parton Distributions (GPDs) and what types of measurements allow access to them. First qualitative simulations using a LO Langevin code demonstrate clearly that different degrees of freedom can behave qualitatively different: the pomeron contributions to the cross sections approach a scaling behavior with a sliding scale dependent on the evolution variable, while the odderon contributions simply are erased in place. This qualitatively different behavior of the odderon contribution under JIMWLK evolution is a genuinely new result. What we find most intriguing, however, is the fact that one can proceed to include new degrees of freedom into the formalism in a consistent manner and that the results are striking. The interplay of group theoretical properties with features imprinted by unresummed perturbation theory have surprisingly strong implications, here the conclusion that the size of the odderon contribution is limited from the outset by consistency of the 


\section{EPJ Web of Conferences}

formalism, not only by phenomenology. We are confident that such features hold also for higher order correlators.

Acknowledgements: Heribert Weigert was supported by NRF under CPRR grant nr 90509 and is grateful for support through labex P2IO during his stay at CPhT Ecole Polytechnique. Tuomas Lappi is supported by Academy of Finland projects 267321 and 273464.

\section{References}

[1] A. Banfi, G. Marchesini, G. Smye, JHEP 08, 006 (2002), hep-ph/0206076

[2] H. Weigert, Nucl. Phys. B685, 321 (2004), hep-ph/0312050

[3] Y. Hatta, JHEP 11, 057 (2008), 0810.0889

[4] S. Caron-Huot (2015), 1501.03754

[5] Y.V. Kovchegov, M.D. Sievert, Phys. Rev. D89, 054035 (2014), 1310.5028

[6] H. Weigert, J. Kuokkanen, K. Rummukainen, AIP Conf. Proc. 1105, 394 (2009)

[7] Y.V. Kovchegov, J. Kuokkanen, K. Rummukainen, H. Weigert, Nucl. Phys. A823, 47 (2009), 0812.3238

[8] C. Marquet, H. Weigert, Nucl. Phys. A843, 68 (2010), 1003.0813

[9] J. Kuokkanen, K. Rummukainen, H. Weigert, Nucl. Phys. A875, 29 (2012), 1108. 1867

[10] Y.V. Kovchegov, L. Szymanowski, S. Wallon, Phys. Lett. B586, 267 (2004), hep-ph/0309281

[11] Y. Hatta, E. Iancu, K. Itakura, L. McLerran, Nucl. Phys. A760, 172 (2005), hep-ph/0501171

[12] N. Kaiser (2009), math-ph/0609082

[13] K. Rummukainen, H. Weigert, Nucl. Phys. A739, 183 (2004), hep-ph/0309306

[14] T. Lappi, H. Mäntysaari, Eur. Phys. J. C73, 2307 (2013), 1212.4825

[15] A. Dumitru, T. Lappi, Y. Nara, Phys. Lett. B734, 7 (2014), 1401.4124 